\title{
Impacto das indicações de procedência no desenvolvimento municipal
}

\author{
Karina Ferreira da Silva Matos ${ }^{1}$ \\ Marcelo José Braga ${ }^{2}$ \\ Pablo Murta Baião Albino ${ }^{3}$
}

Submissão: 25/08/2021

Aceite: $22 / 10 / 2021$

\begin{abstract}
Resumo
As indicações geográficas podem promover o desenvolvimento territorial, por englobar e valorizar diversos aspectos regionais ligados a origem dos produtos, proporcionando ganhos econômicos, a valorização dos produtos e contribuindo para a competitividade da região. $O$ desenvolvimento endógeno territorial utiliza as potencialidades do território englobando aspectos econômicos, ambientais, sociais e políticos, sendo que neste contexto se destaca as relações entre os atores locais em prol da obtenção de objetivos em comum, como o caso da certificação por Indicação de Procedência que não é fornecida de forma individual e isolada. O presente trabalho utilizou para análise especificamente os municípios mineiros que possuem o selo de indicação de procedência fornecido pelo instituto nacional de propriedade industrial. Assim sendo, o presente artigo tem por objetivo: "Mensurar o impacto da certificação de alimentos por Indicação de Procedência no desenvolvimento dos municípios mineiros". Para a realização desta proposta utilizou-se a técnica estatística propensity score matching (PSM). As variáveis de análise foram as seguintes: valor adicionado bruto dos setores de administração, serviços, agropecuária e indústria. Estas variáveis foram obtidas no banco de dados do sidra (IBGE) e são referentes aos municípios mineiros no ano de 2017. Também se utilizou a variável: valor geral do Índice FIRJAN de desenvolvimento municipal. Para a realização deste procedimento utilizou-se o software de análise de dados Stata ${ }^{\circledR}$. Os resultados apontam que o fato dos municípios possuírem algum tipo de Indicação de Procedência em seu território não proporcionou uma diferença significativa no desenvolvimento municipal, quando comparado com municípios que não possuem Indicação de Procedência.
\end{abstract}

Palavras-chave: Indicação de Procedência; Desenvolvimento territorial; Certificação.

\section{Impact of indications of procedence on municipal development}

\begin{abstract}
Geographical indications can promote territorial development, as it encompasses and enhances various regional aspects linked to the origin of products, providing economic gains, the appreciation of products and contributing to the competitiveness of the region. The endogenous territorial development uses the potential of the territory encompassing economic, environmental, social and political aspects, and in this context the relations between the local actors in favor of achieving common goals are highlighted, such as the case of certification by Indication of Origin that it is not provided individually and in isolation. This work used for analysis specifically the municipalities of Minas Gerais that have the seal of indication of origin provided by the national institute of industrial property. Therefore, this article aims to: "Measure the impact of food certification by Indication of Origin on the development of Minas Gerais municipalities". For the realization of this proposal, the statistical technique propensity score matching (PSM) was used. The analysis variables were as follows: gross added value of the sectors of administration, services, agriculture and industry. These variables were obtained from the cider database (IBGE) and refer to the municipalities of Minas Gerais in the year 2017. The variable: general value of the FIRJAN index of municipal development was also used. To perform this procedure, the data analysis software Stata ${ }^{\circledR}$ was used. The results show that the fact that the municipalities have some type of Indication of Origin in their territory did not provide a significant difference in municipal development, when compared with municipalities that do not have Indication of Origin.
\end{abstract}

Keywords: Indication of Origin; Territorial development; Certification.

\footnotetext{
${ }^{1}$ Doutoranda em Extensão Rural (UFV). Email: karinaferreiramatos2019@gmail.com. .

${ }^{2}$ Doutorado em Economia Rural (UFV). Pós-Doutorado (University Of California, UCD, EUA). Professor do Instituto de Políticas Públicas e Desenvolvimento Sustentado (IPPDS) da UFV. Email: mjbraga@ufv.br

${ }^{3}$ Doutorado em Sistemas Flexíveis de Gestão (Universidad Pública de Navarra - UPNA). Email: pablo.albino@ufv.br
} 


\section{Introdução}

Os produtos agropecuários estão presentes no cotidiano da maioria das pessoas e muitas vezes definem características locais e culturais das regiões onde são produzidos. A percepção da importância da ligação entre os produtos agropecuários e seu local de origem é originária dos países europeus que buscavam proteger os seus produtos do uso indevido do nome e concorrência desleal (MARINS; CABRAL, 2015).

Atualmente esta ligação (produto/local de origem) tornou-se ainda mais relevante em decorrência da constante valorização da qualidade dos produtos e apreciação de características singulares dos produtos. Neste contexto, tem-se as indicações geográficas, que são uma forma legal de autenticar a existência da relação entre os produtos e os locais de origem.

As indicações geográficas (IGs) são frutos de processos que averiguam a relação existente entre o produto final e a região de produção, sendo que neste processo estão englobados os aspectos da matéria prima, as relações sociais e a forma de produção. Desta forma, as IGs tendem a possuir uma relação com o desenvolvimento local pois interferem em diversos aspectos (econômicos e sociais) locais.

As IGs tendem a contribuir para a valorização dos produtos e para a estabelecimento de vantagens competitivas dados os fatores de singularidade, diferenciação e aproveitamento de ativos intangíveis relacionados ao local de produção (NIEDERLE, 2009). Contudo, existem autores como, por exemplo, Froehlich, et al. 2010 e Contini, et al. 2016 que apresentam argumentos que se contrapõe a relação positiva entre IGs e desenvolvimento. Os referidos autores argumentam que a IGs proporcionam um processo excludente e que na maioria das vezes beneficia apenas a elite local, não ocasionando o desenvolvimento local esperado.

O desenvolvimento endógeno baseia-se na análise interna dos fatores sociais, políticos e econômicos, sendo um tema cujo estudo tem se destacado em âmbito regional. Segundo Amaral filho (2001) e Vazquez-Barquero (2007) o desenvolvimento endógeno contribui para a melhora no cenário econômico e distribuição de renda local, sendo que este processo é decorrente de mudanças em âmbito social e estratégias econômicas locais.

Nesse sentido, entende-se que o conceito de desenvolvimento territorial está relacionado ao conceito de desenvolvimento endógeno. Sendo que o desenvolvimento territorial se refere de forma específica a determinado território e que considera e utiliza as potencialidades e limitações do mesmo, para facilitar o processo de desenvolvimento. O desenvolvimento territorial está ligado a capacidade da sociedade organizada de provocar mudanças estruturais 
no contexto social, político, cultural econômico, por meio da utilização efetiva dos recursos presentes da região e da força local que se estabeleceu ao longo da história (DALLABRIDA, 2012; PITTERI; SAES; BRESCIANI, 2015).

Neste trabalho adotou-se a perspectiva de um desenvolvimento endógeno territorial, ou seja, um processo de desenvolvimento que está fundamentado na articulação de diversos atores e no aproveitamento das potencialidades oriundas do território, a fim de propiciar benefícios tanto econômicos como também bem-estar social. Diante do exposto, entende-se que as Indicações Geográficas e o desenvolvimento endógeno territorial possui uma íntima relação. Supõe-se que está relação seja positiva, ou seja, que as IGs contribuem para o desenvolvimento endógeno territorial, principalmente por proporcionarem ganhos econômicos ao mesmo que tempo que valorizam diversos aspectos sociais e ambientais.

Ressalta-se que as IGs possuem dois tipos de selos, o de denominação de origem e o de indicação de procedência. Neste trabalho será abordado especificamente o selo referente a Indicação de Procedência (IP), para a obtenção deste selo é necessário que existam uma profunda relação entre a região de produção e o produto, sendo o território reconhecido pelo processo de fabricação (BRASIL, 1996). Assim, entende-se que a obtenção do referido selo pode contribuir para a continuidade das tradições regionais e também para a valorização dos itens produzidos na região.

Neste sentido, o presente artigo tem por objetivo: "Mensurar o impacto da certificação de alimentos por Indicação de Procedência no desenvolvimento dos municípios mineiros". A escolha dos municípios de Minas Gerais se justifica pela heterogeneidade econômica, sua diversidade social, geográfica e cultural que contribuem para a existência de oito certificações por Indicação de Procedência que abrangem 104 municípios. Na próxima seção será apresentado o referencial teórico referente ao desenvolvimento endógeno territorial. Posteriormente, será apresentada a metodologia; os resultados e discussões e por fim as considerações finais.

\section{Desenvolvimento endógeno territorial}

O desenvolvimento é um tema interdisciplinar, abordado sob as mais diversas perspectivas e que possui muitas adjetivações. Nesse sentido, torna-se importante destacar que está seção aborda o desenvolvimento endógeno numa perspectiva territorial, aproximando conceitos e objetivando a homogeneidade para a análise. 
O conceito de desenvolvimento endógeno emerge em um ambiente fortemente influenciado pela elevada competição de mercado e a necessidade de constantes ajustes nos sistemas produtivos. Nesse contexto, se estabelecem novas dinâmicas de organização nos sistemas produtivos locais. Desta forma, o desenvolvimento endógeno de um território parte de um processo em que a sociedade civil assume um papel de protagonista, que por meio de sua capacidade inovadora promove a transformação da organização social e da economia local, a fim de garantir a obtenção de capital principalmente por meio da agregação de valor à produção (VAZQUEZ-BARQUERO, 2007).

Corroborando com a perspectiva supracitada, entende-se que o desenvolvimento endógeno possibilita um processo de crescimento econômico fundamentado em um sistema de agregação de valor sobre a produção e absorção de oportunidade da região, que tendem a ampliar a renda local (AMARAL FILHO, 2001). O desenvolvimento endógeno se inicia por meio de um processo de mudança, que utiliza o potencial existente no território de maneira a modificar sua estrutura econômica e contribuir para a geração de riquezas e aumento do bem-estar social. Possuindo desta forma, uma relação com a utilização e valorização dos recursos locais, por meio de um sistema efetivo de alocação de recursos e introdução de inovações no mercado, que objetivam garantir a percepção de diferenciação territorial (BRAGA, 2002; ARAUJO, 2014).

Outro aspecto relevante do desenvolvimento endógeno é apresentado por Azevedo Junior et al. (2018) que destacam que ele possui potencial para diminuir as desigualdades de renda locais, pois tende a tornar a região mais competitiva por meio da atuação de atores locais e com a utilização dos recursos naturais de forma sustentável. Neste contexto, Braga (2002, p. 24) contribui com a afirmação anteriormente apresentada ao ressaltar que neste processo acontece "a elevação do local ao espaço preferencial da inserção econômica em uma economia globalizada, substituindo a concorrência entre empresas e entre nações pela concorrência entre localidades".

Outro aspecto apresentado na literatura é a importância atribuída a sociedade local no que tange a sua participação no processo de definição de ocupação do espaço, na união de esforços para obter vantagens competitivas e, também, dos benefícios do crescimento econômico (OLIVEIRA; LIMA, 2003; MARQUES, 2017). Diante do exposto, entende-se ser pertinente abordar os aspectos referentes ao desenvolvimento territorial, que pode contribuir para a melhor compreensão da dinâmica de obtenção e influência de uma certificação nos territórios. O desenvolvimento territorial pode ser compreendido por meio dos diversos fatores sociais, econômicos, políticos e ambientais que compõe a estrutura de determinada região. 
Desta forma, engloba diversas características locais que constituem um processo de desenvolvimento próprio voltado para o aproveitamento das potencialidades territoriais.

Segundo Dallabrida (2012), o desenvolvimento territorial pode ser considerado como sendo um processo no qual a sociedade organizada proporciona uma mudança estrutural no contexto local, objetivando a melhoria da qualidade de vida e dos aspectos econômicos. Nesse sentido, Peres, Müller e Verdi (2006) apontam que o desenvolvimento territorial é um processo complexo que exige a existência de uma articulação de atores sociais, que pautados em diversas estratégias busquem tanto o desenvolvimento produtivo como também social. Segundo os autores supracitados, a capacidade coletiva e de reação são aspectos de suma importância e de grande interferência no processo de desenvolvimento territorial.

Diante do exposto, entende-se que as principais características do desenvolvimento territorial envolvem não apenas as questões econômicas, mas sobretudo a organização social em torno de objetivos em comum, que visam garantir possibilidades diversas para o território. Corroborando com esta perspectiva, diversos autores (BARJOLLE; SYLVANDER, 2002; ALLAIRE et al., 2005; ROCHA, 2010; MACKE; SARATE, 2015; SANZ-CAÑADA; MUCHNIK, 2016; GARCIA et al., 2018) destacam que o desenvolvimento está intimamente relacionado com as interações sociais, o fortalecimento das instituições locais e os fatores históricos de determinada região.

Desta forma, o desenvolvimento territorial seria fruto de uma ação coletiva que englobaria os fatores econômicos, sociais e locais. Diante do exposto, Rocha (2010) ressalta três componentes fundamentais do desenvolvimento territorial, sendo eles:

\footnotetext{
i) as dinâmicas econômicas, sociais, políticas e culturais endógenas ao território; ii) a arquitetura (ou tecido) institucional predominante e a sua influência sobre o modo como as políticas públicas incidem no território; e iii) os processos sociais e os mecanismos institucionais por meio dos quais o território se relaciona com o seu entorno e o 'resto do mundo' (ROCHA, 2010, p.66).
}

No que tange a relação do desenvolvimento territorial com as Indicações Geográficas, destaca-se que o "aumento da demanda pelos produtos frutos da IG, não é apenas um fim, mas um instrumento, um meio para que se atinja o desenvolvimento" (SILVA, BRITO, DANTAS, 2016, p. 2986). Corroborando com esta perspectiva, Barjolle e Sylvander (2002) apontam que os processos de IG tendem a proporcionar impactos positivos nas economias regionais e a promover o desenvolvimento, gerando principalmente benefícios sociais.

Barjolle, Paus e Perret (2009) apresentam três dimensões referentes ao processo de desenvolvimento relacionado com as indicações geográficas, sendo elas: dimensão econômica; a dimensão social; e a dimensão ambiental. Neste contexto os autores ressaltam que as questões 
que mais se destacam são as econômicas. Contudo, ressalvam que para a manutenção do sucesso de uma indicação geográfica os outros aspectos devem ser também valorizados.

Em suma, considerando-se todos os conceitos e aspectos apresentados, entende-se que o desenvolvimento endógeno territorial se fundamenta na efetiva utilização das potencialidades do território, por meio da articulação da sociedade local a fim de proporcionar benefícios econômicos e bem-estar social. Na próxima seção será apresentada a metodologia.

\section{Metodologia}

Este trabalho pode ser classificado como exploratório e descritivo. Exploratório por propiciar mais compreensão sobre o problema em análise. Descritivo por apresentar as características da região estudada (GIL, 2010). Possui uma abordagem quantitativa para a qual utilizou-se a técnica estatística: propensity score matching (PSM). Está técnica foi escolhida por ser capaz de mensurar efetivamente o impacto de uma variável em um determinado contexto, ou seja, esta técnica permite eliminar os vieses dos outros elementos que poderiam interferir nos resultados e considerar apenas a variável de interesse (no caso, o impacto da Indicação de Procedência) (BECKER; ICHINO, 2002).

Diversos trabalhos (FERNANDES; TAVARES; AZEVEDO, 2018; WIDMARCK, 2020; DUARTE; ALVES, 2019; LIMA; MAIA, 2016; MACEDO; PORTO, 2017; OLIVEIRA; DORNER; SHIKIDA, 2017; MACEDO; OLINDA; LEITE, 2015; MOURA; SOUSA, 2020) com diferentes técnicas estatísticas, utilizaram a variável "Valor Adicionado Bruto" para explicar o desenvolvimento local. Dentre estes, algum autores (FERNANDES; TAVARES; AZEVEDO, 2018; WIDMARCK, 2020; DUARTE; ALVES, 2019) também consideraram em suas análises o IFDM (Índice FIRJAN de Desenvolvimento Municipal). Neste sentido, entende-se ser pertinente utilizar estas variáveis para a realização da técnica proposta, pois existe um respaldo na literatura e encontrou-se dados mais recentes referentes a estas variáveis, o que corrobora para uma análise atualizada.

A técnica propensity score matching (PSM) consiste em "identificar unidades não-tratadas que sejam similares às unidades tratadas e comparar as médias no resultado procurado entre estes dois grupos para identificar o impacto do tratamento" (RAMOS, 2009, p. 106). Segundo Rosenbaum e Rubin (1983) a equação da técnica propensity score matching é a seguinte:

$$
\left(Y_{1}^{i}, Y_{0}^{i} \perp D_{i}\right) \mid Z_{i}, \text { então }\left(Y_{1}^{i}, Y_{0}^{i} \perp D_{i}\right) \mid \operatorname{Pr}\left(Z_{i}\right), \text { ondePr }\left(Z_{i}\right)=\operatorname{Pr}
$$


Desta forma, tem-se que a técnica analisa a variável dependente $Y$ binária ( $O$ ou 1 ) em um conjunto específico $Z_{i}$ (variáveis independentes), onde obtém-se o resultado referente a probabilidade de $\operatorname{Pr}\left(Z_{i}\right)$ participar do grupo 1.

$$
\Delta=E
$$

Em que:

$Y_{0}^{i}$ é a variável dependente $Y$ para cada município i dado o fato de não possuir Indicação de Procedência.

$Y_{1}^{i}$ é a variável dependente $Y$ para cada município i dado o fato de possuir Indicação de Procedência.

$Z_{i}$ é o conjunto de variáveis observadas.

Diante do exposto, para a utilização da técnica propensity score matching (PSM) neste trabalho, foram utilizadas as variáveis: valor adicionado bruto da administração (mil reais); valor adicionado bruto dos serviços (mil reais); valor adicionado bruto da agropecuária (mil reais); e valor adicionado bruto da indústria (mil reais). Estas variáveis foram obtidas no banco de dados do SIDRA (IBGE) e são referentes aos municípios mineiros no ano de 2017. Também utilizou-se a variável: valor geral do Índice FIRJAN de Desenvolvimento Municipal (edição 2018, ano base 2016), este indicador engloba diferentes áreas sociais (emprego e renda, saúde e educação, que também foram considerados separadamente), variando em uma escala de 0 a 1 , onde quanto mais próximo de 1 melhor o desenvolvimento. Para a realização deste procedimento utilizou-se o software de análise de dados STATA ${ }^{\circledR}$.

\section{Resultados e discussões}

4.1 Descrição dos dados

A análise descritiva dos dados está apresentada na tabela 1, sendo que os valores foram calculados em formato nominal. Analisando-se as variáveis "Valor Adicionado Bruto Agropecuária", "Valor Adicionado Bruto - Indústria", "Valor Adicionado Bruto - Serviços", "Valor Adicionado Bruto - Administração", observa-se que em todas elas a média de valores está mais próxima do valor mínimo, o que indica que existem muitas observações perto do valor mínimo. Contudo, a amplitude, ou seja, a diferença entre o valor máximo e o mínimo, nestas variáveis é considerada alta, indicando que existem observações cujos valores das variáveis são significativamente altos. Neste sentido, considerando-se os municípios, entende-se que a realidade destes se apresenta de maneira discrepante e que a maioria dos municípios possui uma realidade mais próxima dos valores mínimos das variáveis analisadas. 
Em relação as variáveis "IFDM Geral", "IFDM Emprego e Renda", "IFDM Educação" e "IFDM Saúde", observa-se que a variável "IFDM Emprego e Renda" possui a maior amplitude e que o valor médio está mais próximo do valor mínimo, o que indica que existem muitas observações perto do valor mínimo. Este fato vai ao encontro da análise das variáveis relacionadas ao valor adicionado bruto e corrobora a ideia de que o cenário econômico destes municípios está em sua maioria mais próximo dos valores mínimos.

Tabela 1 - Valor adicionado bruto dos setores

\begin{tabular}{|c|c|c|c|c|c|}
\hline Variável & Observações & Média & Desvio Padrão & Mínimo & Máximo \\
\hline $\begin{array}{l}\text { Valor Adicionado Bruto } \\
\text { Agropecuária }\end{array}$ & 853 & 33659,45 & 64256,75 & 46 & 784149 \\
\hline $\begin{array}{l}\text { Valor Adicionado Bruto } \\
\text { Indústria }\end{array}$ & 853 & 150500,1 & 697045,2 & 539 & $\begin{array}{l}1,08 \\
x 10^{07}\end{array}$ \\
\hline $\begin{array}{l}\text { Valor Adicionado Bruto } \\
\text { Serviços }\end{array}$ & 853 & 302147,1 & 2099890 & 4595 & $\begin{array}{l}5,44 \\
\times 10^{07} \\
\end{array}$ \\
\hline $\begin{array}{l}\text { Valor Adicionado Bruto } \\
\text { Administração }\end{array}$ & 853 & 105603,2 & 446998,6 & 8842 & $\begin{array}{l}1,14 \\
\times 10^{07} \\
\end{array}$ \\
\hline IFDM geral & 842 & 0,67777597 & 0,0675452 & 0,456849 & 0,858649 \\
\hline Indicação de procedência & 853 & 0,116061 & 0,3204859 & 0 & 1 \\
\hline IFDM emprego e renda & 842 & 0,4470897 & 0,1104011 & 0,190595 & 0,795521 \\
\hline IFDM educação & 853 & 0,8134129 & 0,0529149 & 0,602521 & 0,979912 \\
\hline IFDM saúde & 853 & 0,7726072 & 0,1091481 & 0,356905 & 0,972298 \\
\hline
\end{tabular}

Fonte: Dados da pesquisa

\subsection{Propensity score matching (PSM)}

A técnica PSM aplicada obteve balanceamento satisfatório. A tabela 2 apresenta a distribuição dos quatro grupos gerados pelo PSM, sendo que o "0" representa o grupo controle, ou seja, sem indicação de procedência. Já o grupo " 1 " indica o grupo tratado, ou seja, com indicação de procedência.

Tabela 2 - Distribuição dos grupos

\begin{tabular}{llll}
\hline Distribuição & 0 & 1 & Total \\
\hline 0,0040152 & 631 & 44 & 675 \\
\hline 0,2 & 70 & 20 & 90 \\
\hline 0,3 & 29 & 18 & 47 \\
\hline 0,4 & 6 & 17 & 23 \\
\hline Total & 736 & 99 & 835
\end{tabular}

Fonte: Dados da pesquisa

A tabela 3 contém o resultado do Average Treatment effect on the Treated (ATT), ou seja, a indicação da estatística com a média do efeito de tratamento no indivíduo tratado. Em outras 
palavras este resultado demonstra se há diferença estatística em relação ao impacto de se ter ou não uma indicação de procedência. Nos resultados apresentados, observa-se que a estatística t possui o valor de "-0,424", sendo que valores abaixo de 1,645 não são significantes a nível de 10\%. Ressalva-se que o modelo utilizado foi o de pareamento do vizinho mais próximo.

Tabela 3 - Average Treatment effect on the Treated

\begin{tabular}{lllll}
\hline Número tratado & Número de controle & ATT & Erro Padrão & Estatística t \\
\hline 99 & 75 & $-0,005$ & 0,011 & $-0,424$ \\
\hline
\end{tabular}

Fonte: Dados da pesquisa

A tabela 4 demonstra que quando foi analisado a diferença de médias para os grupos sem pareamento (IFDM sem pareamento) identificou-se que havia diferença estatisticamente significante. Porém, após retirar os vieses a partir do pareamento das amostras, nota-se que a diferença estatística entre os grupos tratados e de controle não se apresentaram significativamente diferentes entre os grupos. Em resumo, o fato de ter indicação geográfica não corresponde há uma diferença no nível de desenvolvimento entre os municípios.

Tabela 4 - Diferenças das médias sem pareamento

\begin{tabular}{llrrrrr}
\hline Variável & Tipo de amostra & Tratado & Controle & Diferença & Erro padrão & Estatística t \\
\hline IFDM & Sem pareamento & 0,716317453 & 0,672622109 & 0,4369534 & 0,007072066 & 6,18 \\
\hline & ATT & 0,713273978 & 0,715448372 & $-0,0021743$ & 0,010464694 & $-0,21$
\end{tabular}

Fonte: Dados da pesquisa

A tabela 5 demonstra que quando analisado pelas três dimensões que compõe o índice de desenvolvimento os resultados se mostraram semelhantes ao índice de desenvolvimento geral, não se mostrando significativos.

Tabela 5 -Dimensões que compõe o índice de desenvolvimento

\begin{tabular}{llrrrrr}
\hline Variável & Tipo de amostra & Tratado & Controle & Diferença & Erro padrão & Estatística t \\
\hline $\begin{array}{l}\text { Emprego e } \\
\text { Renda }\end{array}$ & Sem pareamento & 0,515436343 & 0,437982945 & 0,077453398 & 0,011512766 & 6,73 \\
\hline & & & & & \\
\hline Educação & STT & 0,498136177 & 0,521894679 & $-0,02375850$ & 0,01831618 & $-1,30$ \\
\hline & STT pareamento & 0,832339231 & 0,81092785 & 0,2141138 & 0,005612047 & 3,82 \\
\hline Saúde & 0,829361921 & 0,82176242 & 0,007599501 & 0,009159347 & 0,83 \\
\hline & Sem pareamento & 0,8011768 & 0,768874036 & 0,032302763 & 0,011650756 & 2,77 \\
\hline
\end{tabular}

Fonte: Dados da pesquisa

Em suma, observa-se que não houve uma diferença estatística entre os municípios que possuem e os que não possuem indicação de procedencia, logo entende-se que não há impacto das indicações de procedência no desenvolvimento municipal. Contudo, esta análise aponta para a direção contrária a maioria da literatura consultada. Sendo assim, considera-se que outros 
fatores podem estar ocasionando este cenário como, por exemplo, a pouca ou nenhuma capacitação administrativa dos agricultores (principalmente os que possuem pequenas propriedades) para aproveitar os benefícios da certificação (BATALHA; BUAINAIN; SOUZA FILHO, 2005; MAZZOLENI; NOGUEIRA, 2006); um cenário político que não proporcione e nem incentive a valorização da cultura local e do turismo (BENI, 1999; CRUZ, 2005); uma concentração de benefícios nas mãos da elite produtora que deseja manter o status quo (FROEHLICH, ET AL. 2010; CONTINI, ET AL. 2016).

\section{Considerações finais}

O presente estudo teve por objetivo mensurar o impacto da certificação de alimentos por Indicação de Procedência no desenvolvimento dos municípios mineiros. Diante dos resultados encontrados, percebe-se que o fato dos municípios possuírem algum tipo de Indicação de Procedência em seu território não proporcionou uma diferença significativa no desenvolvimento municipal, quando comparado com municípios que não possuem Indicação de Procedência. Desta forma, entende-se que não houve impacto da Indicação de Procedência no desenvolvimento municipal.

Esse resultado evidencia uma possível fragilidade no processo de gestão entorno dos benefícios que uma Indicação de Procedência pode proporcionar a todos os produtores dos municípios envolvidos no processo de certificação. Também demonstra a necessidade de políticas públicas que auxiliem a população local, principalmente em termos administrativos, incluindo questões como, gestão, logística e marketing. Desta forma, os produtores aproveitariam não apenas os ganhos oriundos dos produtos em si, mas também se beneficiariam de todo contexto e entorno local como, por exemplo, com o turismo gastronômico.

Para estudos futuros sugere-se que se adote técnicas qualitativas, especificamente entrevistas em profundidade, a fim de compreender se existem benefícios intrínsecos e não financeiros no fato de se possuir uma Indicação de Procedência e que não podem ser percebíveis com dados quantitativos.

\section{Referências}

ALLAIRE, G. et al. Les dispositifs français et européens de protection de la qualité et de l'origine dans le contexte de l'OMC: justifications générales et contextes nationaux. p. 1-21, 2005. In: 
Symposium international Territoires et enjeux du développement régional. Lyon: CIHEAMIAMM. 2005.

AMARAL FILHO, J. D. A endogeneização no desenvolvimento econômico regional e local. Planejamento e políticas públicas, n. 23, p.261-286, 2001.

ARAUJO, R. D. C. D. Análise sobre a monocultura de soja e o Desenvolvimento Sustentável na Amazônia com base na teoria do Desenvolvimento Endógeno. Economia e Desenvolvimento, v. 26, n. 1, p.105-118, 2014.

AZEVEDO JUNIOR, W. C. D. et al. Potencial da acrocomia aculeata no desenvolvimento endógeno da região de Cuiabá, Brasil. Revibec: revista iberoamericana de economía ecológica, v. 28, p. 52-65, 2018.

BARJOLLE, D., PAUS, M.; PERRET A. Impacts of Geographical Indications Review of Methods and Empirical Evidence. Agricultural Economists Conference, Beijing, China, August 16-22, 2009.

BARJOLLE, D.; SYLVANDER, B. Some factors of success for origin labelled products in agri-food supply chains in Europe: market, internal resources and institutions. Economies et sociétés, v. 25, p. 1-31, 2002.

BATALHA, M. O.; BUAINAIN, A. M.; SOUZA FILHO, H. M. D. Tecnologia de gestão e agricultura familiar. Gestão Integrada da Agricultura Familiar. São Carlos (Brasil): EDUFSCAR, p. 43-66, 2005.

BECKER, Sascha O.; ICHINO, Andrea. Estimation of average treatment effects based on propensity scores. The stata journal, v. 2, n. 4, p. 358-377, 2002.

BENI, M. C. Política e estratégia do desenvolvimento regional: planejamento integrado e sustentável do turismo. Revista Turismo em análise, v. 10, n. 1, p. 7-17, 1999.

BRAGA, T. M. Desenvolvimento local endógeno: entre a competitividade e a cidadania. Revista Brasileira de Estudos Urbanos e Regionais, n. 5, p. 23-37, 2002.

BRASIL. Lei no 9.279/96, Regula direitos e obrigações relativos à propriedade industrial. 1996. Disponível em:< http://www.planalto.gov.br/ccivil_03/leis/l9279.htm>. Acesso em: 07 de fev. de 2020.

CONTINI, C. et al. Why do we buy traditional foods? Journal of Food Products Marketing, v. 22, n. 6, p. 643-657, 2016.

CRUZ, R. D. C. A. D. Políticas públicas de turismo no Brasil: território usado, território negligenciado. Geosul, v. 20, n. 40, p. 27-43, 2005.

DALLABRIDA, V. R. Território e desenvolvimento sustentável: Indicação Geográfica da ervamate de ervais nativos no Brasil. Informe Gepec, v. 16, n. 1, p. 42-59, 2012.

DUARTE, V. N.; ALVES, L. R. Índice firjan de desenvolvimento municipal no estado de mato grosso do sul: o que mostram os números? X Seminário Internacional sobre Desenvolvimento Regional, 2019.

FERNANDES, R. B.; TAVARES, A. D. L.; AZEVEDO, Y. G. P. Relação entre o valor adicionado das atividades econômicas e o produto interno bruto do Rio Grande do Norte. Race: revista de administração, contabilidade e economia, v. 17, n. 2, p. 757-782, 2018.

FROEHLICH, J. M. et al. A agricultura familiar e as experiências de Indicações Geográficas no Brasil meridional. Agrociencia Uruguay, v. 14, n. 2, p. 115-125, 2010. 
GARCIA, D. L. et al. Indicação Geográfica e o Desenvolvimento Regional do Baixo São Francisco Sergipano. Revista INGI-Indicação Geográfica e Inovação, v. 2, n. 2, p. 80-94, 2018.

GIL, A. C. Como elaborar projetos de pesquisa. 5 ed. São Paulo: Atlas, 2010. 184 p.

INPI. Instrução Normativa № 25/2013, Condições para o Registro das Indicações Geográficas. Instituto Nacional da Propriedade Industrial. 2013. Disponível em:

<http://www.inpi.gov.br/legislacao-1/in_25_21_de_agosto_de_2013.pdf>. Acesso em: 07 de fev. de 2020.

LIMA, V. M. A. D.; MAIA, K. Índice de desenvolvimento socioeconômico dos municípios sulmato-grossenses para 2010. Revista Brasileira de Economia de Empresas, v. 15, n. 2, 2016.

MACEDO, F. C. D.; PORTO, L. R. Dinâmica regional brasileira: uma análise com base nos dados do pib municipal (2002-2015). Revista de Economia Regional, Urbana e do Trabalho, v. 6, n. 2, p. 52-69, 2017.

MACEDO, L. O. B.; OLINDA, R. A. D.; LEITE, F. N. D. S. Elaboração de um indicador de atividade econômica municipal para a análise de conjuntura econômica do Município de RondonópolisMT. Revista de Administração e Negócios da Amazônia, v. 7, n. 2, p. 159-178, 2015.

MACKE, J.; SARATE, J. A. R. Desenvolvimento territorial e capital social: elementos, conexões e proposta de avaliação de territórios. Revista Brasileira de Gestão e Desenvolvimento Regional, v. 11, n. 3, p.56-79, 2015.

MARINS, M. F.; CABRAL, D. H. Q. O papel da Indicação Geográfica como propulsor da inovação e do desenvolvimento local: caso Vale dos Vinhedos. Cadernos de Prospecção, v. 8, n. 2, p. 406414, 2015.

MARQUES, A. P. Os caminhos sinuosos do desenvolvimento. Desenvolvimento e Sociedade, v. 2, n. 2, p. 31-49, 2017.

MAZZOLENI, E. M.; NOGUEIRA, J. M. Agricultura orgânica: características básicas do seu produtor. Revista de Economia e Sociologia Rural, v. 44, n. 2, p. 263-293, 2006.

MELO, C. O. D.; PARRÉ, J. L. Índice de desenvolvimento rural dos municípios paranaenses: determinantes e hierarquização. Revista de Economia e Sociologia Rural, v. 45, n. 2, p. 329365, 2007.

MOURA, J. E. A.; SOUSA, E. P. D. Análise multidimensional do desenvolvimento rural nos municípios cearenses e pernambucanos. Geosul, v. 35, n. 76, p. 706-730, 2020.

OLIVEIRA, G. B. D.; LIMA, J. E. D. S. Elementos endógenos do desenvolvimento regional: considerações sobre o papel da sociedade local no processo de desenvolvimento sustentável.

Revista da FAE, v. 6, n. 2, p. 29-37, 2003.

OLIVEIRA, T. J. A. D.; DORNER, S. H.; SHIKIDA, P. F. A. A agricultura familiar e o desenvolvimento rural no nordeste do Brasil: uma análise comparativa com a região sul. Acta Tecnológica, v. 10, n. 2, p. 59-74, 2017.

PERES, E. L. D. S.; MÜLLER, G.; VERDI, A. R. Instituições, territórios e desenvolvimento local: delineamento preliminar dos aspectos teóricos e morfológicos. Geografia, v. 31, n. 3, p. 437454, 2006.

RAMOS, M. Aspectos conceituais e metodológicos da avaliação de políticas e programas sociais. Planejamento e políticas públicas, v. 1, n. 32, 2009.

ROCHA, J. D. A importância do capital institucional na sustentabilidade do desenvolvimento territorial. Sustentabilidade em Debate, v. 1, n. 1, p. 63-78, 2010. 
ROSENBAUM, P. R.; RUBIN, D. B. The central role of the propensity score in observational studies for causal effects. Biometrika, v. 70, n. 1, p. 41-55, 1983.

SANZ-CAÑADA, J.; MUCHNIK, J. Geographies of origin and proximity: Approaches to local agrofood systems. Culture \& History Digital Journal, v. 5, n. 1, p. 1-19, 2016.

SILVA, C. K. V.; BRITO, L. M.; DANTAS, T. K. D. S. A indicação geográfica como promotora do desenvolvimento local e regional: o caso (em potencial) do bordado do Seridó. Revista GEINTEC-Gestão, Inovação e Tecnologias, v. 6, n. 1, p. 2982-2990, 2016.

VAZQUEZ-BARQUERO, Antonio. Desarrollo endógeno. Teorías e políticas de desarrollo terrotorial. Investigaciones Regionales, n. 11, p. 183-210, 2007.

WIDMARCK, J. D. J. A. Em busca do desenvolvimento territorial rural do Matopiba: uma análise do Cerrado. Revista Economia Ensaios, v. 35, n. 2, 2020. 